TITLE:

\title{
Direct investigations of the interface impedance of organic field-effect transistors with self- assembled-monolayer-modified electrodes
}

\section{$\operatorname{AUTHOR}(\mathrm{S})$ :}

Kimura, Tomoharu; Kobayashi, Kei; Yamada, Hirofumi

\section{CITATION:}

Kimura, Tomoharu ... [et al]. Direct investigations of the interface impedance of organic field-effect transistors with self-assembled-monolayer-modified electrodes. Organic Electronics: physics, materials, applications 2016, 38: 74-78

\section{ISSUE DATE:}

2016-11

URL:

http://hdl.handle.net/2433/216684

\section{RIGHT:}

(C) 2016. This manuscript version is made available under the CC-BY-NC-ND 4.0 license

http://creativecommons.org/licenses/by-nc-nd/4.0/; The full-text file will be made open to the public on 01 Novembe 2018 in accordance with publisher's 'Terms and Conditions for Self-Archiving'.; この論文は出版社版でありません。引 用の際には出版社版をご確認ご利用ください。; This is not the published version. Please cite only the published version. 


\title{
Direct investigations of the interface impedance of organic field-effect transistors with self-assembled-monolayer-modified electrodes
}

\author{
Tomoharu Kimura $^{\text {a, }}{ }^{*}$, Kei Kobayashi ${ }^{\text {a,b }}$, Hirofumi Yamada ${ }^{\text {a }}$ \\ ${ }^{a}$ Department of Electronic Science and Engineering, Kyoto University, Kyoto, 615-8510, Japan \\ ${ }^{\mathrm{b}}$ The Hakubi Center for Advanced Research, Kyoto University, Kyoto, 615-8520, Japan
}

\begin{abstract}
It has been demonstrated that the modification of electrodes with self-assembled monolayers (SAMs) reduces the contact resistance and improves the device performances of organic field-effect transistors (OFETs). However, it has been difficult to judge if the contact resistance was reduced by the change in the electronic properties or by the change in the morphology of the metal-organic interface caused by the SAM modification because they have been difficult to be separately assessed. We have directly investigated the local impedance and the potential difference at the electrode-channel interfaces of the OFETs with and without modification of the electrodes by a pentafluorobenzenethiol SAM using frequency-modulation scanning impedance microscopy (FM-SIM). The potential profile measurement and the FM-SIM measurement at the interface showed that the improvement of the field-effect mobility in the SAM-modified OFET was caused by the reduction of the energy level mismatch, namely, the hole injection barrier at the source-channel interface, presumably with the reduction of the hole trap sites at the source-channel interface.
\end{abstract}

Keywords: Organic field-effect transistor, Atomic force microscopy, Self-assembled monolayer, Metal-organic interface

\footnotetext{
* Corresponding author

E-mail address: t.kimura@piezo.kuee.kyoto-u.ac.jp (T. Kimura).
} 


\section{Introduction}

Organic field-effect transistors (OFETs) have currently been widely studied for their potential applications to light-weight and flexible electronic devices [1,2]. The performance of the OFETs, however, is still not sufficient for practical applications partly because they are limited by the electronic properties at the non-ideal metal-organic interfaces $[3,4]$. Such metal-organic interfaces not only exhibit high contact resistances, but also cause non-linearities in the current-voltage characteristics. The dependence of the field-effect mobility on the gate bias voltages is also caused by a non-ideal metal-organic interface, which makes it difficult to control the device performances. The influence of the metal-organic interfaces on the electronic properties becomes a major issue that limits the device performances of the OFETs, especially of those having a short channel length and those using organic molecules exhibiting a high carrier mobility $[5,6]$.

Recent studies showed that modification of the electrodes with self-assembled monolayers (SAMs) reduces the contact resistances and thereby improves the device performances of the bottom-contact in the OFETs [7-11]. The contact resistances at the metal-organic interfaces correlate with the carrier injection barrier induced by an energy level mismatch between the Fermi energy $\left(E_{\mathrm{F}}\right)$ of the metal and the highest occupied molecular orbital (HOMO) energy $\left(E_{\mathrm{HOMO}}\right)$ of the organic film. Thus, the use of SAMs having negative electronic dipoles increases the work function of the electrodes, which results in the reduction of the carrier injection barrier at the metal-organic interfaces $[8,9,12]$. Another report, however, showed a greater reduction in the contact resistance of the OFETs with SAM-modified electrodes, despite the fact that the modified electrode showed almost the same work function as that of bare electrodes [10]. Usually SAMs not only modify the surface energy of the metal electrodes, but also affect the crystallinity and grain sizes of the organic thin films [11]. While the performance can be improved by changing the electronic properties or by a change in the morphology of the metal-organic interface caused by the SAM-modification, they have been difficult to be separately assessed by conventional measurements using large-area films and electrodes like the transition line method [13] or photoelectron spectroscopy [9].

We recently developed frequency-modulation scanning impedance microscopy (FM-SIM) that can sensitively measure not only the potential differences, but also the local impedances at the metal- 
organic interface [14]. In a previous paper [14], we investigated the impedance of a Pt electrode and a single pentacene grain by FM-SIM and revealed that the reduction of the contact resistance at the interface by the voltage application was caused by the reduction of the energy level mismatch. In the present study, the electrodes of OFETs having dinaphtho[2,3-b:2'3'-f]thieno[3,2-b]thiophene (DNTT) active layers are modified by pentafluorobenzenethiol (PFBT), which is a thiol-based SAM material widely used in small molecule OFETs $[15,16]$. We first characterized the electrical characteristics of the OFETs with SAM-modified and bare electrodes, and directly investigated the potential difference and the local impedance at the electrode-channel interfaces of the operating OFETs by the FM-SIM. We then discuss the mechanisms for improving the device performance in the SAM-modified OFET in terms of the change in the interface impedance as a function of the gate bias voltage.

\section{Experimental}

We fabricated bottom-contact OFET devices of DNTT with and without the electrodes modified by PFBT-SAM. The schematic of the DNTT-OFET with the PFBT-SAM-modified Au electrodes, and chemical structures of the DNTT and PFBT, are shown in Fig. 1(a). Twenty-nm-thick Au electrodes, whose channel width and channel length were approximately $1 \mu \mathrm{m}$ and $500 \mathrm{~nm}$, respectively, were fabricated by photolithography on a heavily doped n-type Si substrate with a 100-nm-thick thermally grown oxide layer. The PFBT-SAM was formed by immersing the Si substrate with Au electrodes in a $30 \mathrm{mM}$ isopropanol solution of PFBT for $5 \mathrm{~min}$ followed by a rinse with isopropanol. A 100-nm-thick active layer of DNTT was deposited on the substrates with (PFBT-Au) and without SAM modification (bare-Au). The transfer characteristics of the DNTT-OFETs were obtained using the Keithley 4200 semiconductor characterization system. 
(a)

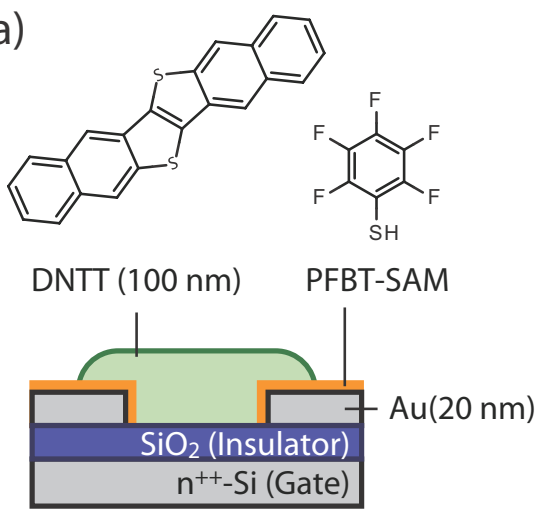

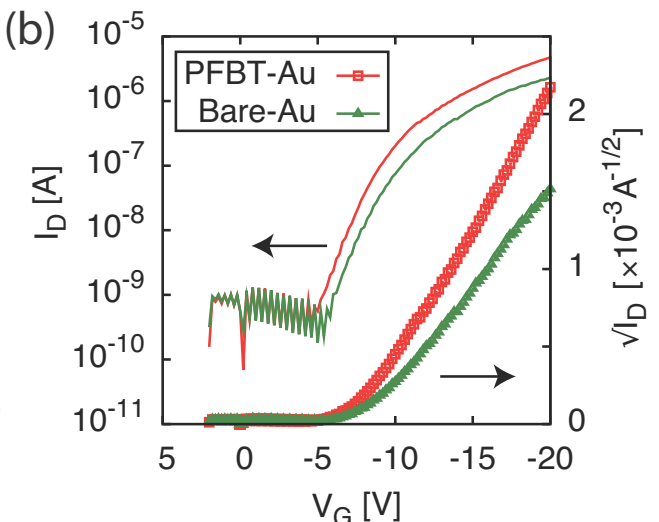

Fig. 1. (a) Chemical structures of DNTT and PFBT, and schematic of DNTT-OFET with PFBT-SAM-modified $\mathrm{Au}$ electrodes. (b) Transfer characteristics of DNTT-OFETs with PFBT-SAM-modified and bare Au electrodes.

The FM-SIM measurements were performed on both OFETs. Figure 2 shows the schematic diagram of the FM-SIM measurement setup. The cantilever was oscillated at its resonance frequency and the constant tip-sample distance was performed by keeping the frequency shift of the cantilever $(\Delta f)$ constant, that is, frequency-modulation atomic force microscopy (FM-AFM). The local potential $V_{\mathrm{lo}}{ }^{\mathrm{dc}}$ was measured by nullifying the $f_{\mathrm{t}}$ component in the $\Delta f$ signal induced by an ac bias voltage applied to the cantilever (frequency: $f_{\mathrm{t}}=1 \mathrm{kHz}$, amplitude: $V_{\mathrm{t}}^{\mathrm{ac}}=2 \mathrm{~V}_{\mathrm{p}-\mathrm{p}}$ ), that is, Kelvin-probe force microscopy (KFM) [17]. At the same time, another ac bias voltage was applied to the source electrode (frequency: $f_{\mathrm{s}}=100 \mathrm{~Hz}$, amplitude: $V_{\mathrm{s}}^{\text {ac }}=1 \mathrm{~V}_{\mathrm{p}-\mathrm{p}}$ ). We now describe the variation in the surface potential under the tip induced by $V_{\mathrm{s}}^{\text {ac }}$ as $V_{\mathrm{lo}}^{\mathrm{ac}} \cos \left(2 \pi f_{\mathrm{s}} t+\phi_{\mathrm{lo}}\right)$. Since the electrostatic force exerted on the cantilever tip is proportional to the square of the local voltage, the frequency component at $f_{\mathrm{s}}+f_{\mathrm{t}}$ in the $\Delta f$ signal, called the "FM-SIM signal", is given by

$$
\Delta f_{\mathrm{s}+\mathrm{t}}=\alpha V_{\mathrm{lo}}^{\mathrm{ac}} \cos \left[2 \pi\left(f_{\mathrm{s}}+f_{\mathrm{t}}\right) t+\phi_{\mathrm{lo}}\right]
$$

where $\alpha$ is a proportional factor, which is constant while the tip-sample distance is kept constant. By measuring the amplitude and phase of the $\Delta f_{\mathrm{s}+\mathrm{t}}$ component, we can calculate $V_{\mathrm{lo}}^{\text {ac }}$, from which we can evaluate the interface impedance using the appropriate circuit model. Thus, we can visualize the variation in the interface impedance [14]. 
We performed the FM-SIM measurements using a commercially available AFM apparatus (JEOL: JSPM-4200) with a lab-built AFM controller and a Pt-coated conductive cantilever (Olympus: OMCL-AC240TM-R3), whose nominal spring constant and resonance frequency were $2 \mathrm{~N} / \mathrm{m}$ and 70 $\mathrm{kHz}$, respectively. The $\Delta f$ signal was detected by a lab-built FM detector [18], and the demodulation of the frequency modulation in the $\Delta \mathrm{f}$ signal were performed by lock-in amplifiers (NF Corporation: LI5640 and Zurich Instruments: HF2LI-MF). All of the measurements were performed at room temperature and under a vacuum condition $\left(<1 \times 10^{-3} \mathrm{~Pa}\right)$.

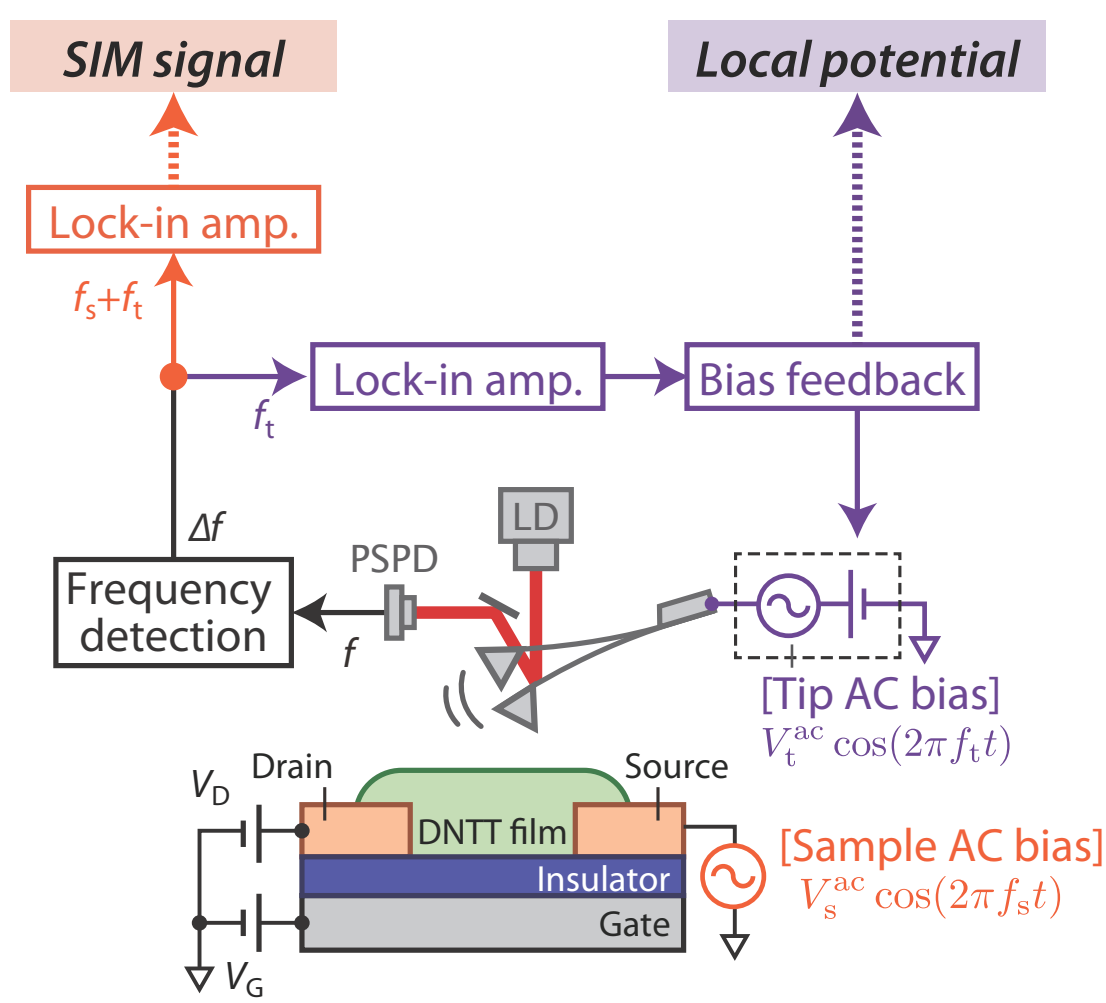

Fig. 2. Schematic diagram of FM-SIM measurement setup. The conductive cantilever is oscillated at its resonance frequency, and the topography is obtained by keeping the tip-sample distance constant (FM-AFM). Two ac bias voltages are applied to the cantilever $\left(V_{\mathrm{t}}^{\mathrm{ac}} \cos \left(2 \pi f_{\mathrm{t}} t\right)\right)$ and the source electrode $\left(V_{\mathrm{s}}^{\mathrm{ac}} \cos \left(2 \pi f_{\mathrm{s}} t\right)\right)$. The $f_{\mathrm{t}}$ component in the resonance frequency shift $(\Delta f)$ signal is detected and then nullified to measure the local surface potential, while the $f_{\mathrm{s}}+f_{\mathrm{t}}$ component in the $\Delta f$ signal is detected as the "FM-SIM signal", corresponding to the local ac voltage on the sample. 


\section{Results and Discussion}

The transfer characteristics of the DNTT-OFETs with and without SAM modification are shown in Fig. 1(b). The current of the PFBT-Au device was apparently greater than that of the OFET with bare-Au electrodes. The field-effect mobility of the PFBT-Au device, averaged for three devices on the same substrate, was $0.99 \mathrm{~cm}^{2} \mathrm{~V}^{-1} \mathrm{~s}^{-1}$, which was more than twice that of the bare-Au device $(0.44$ $\left.\mathrm{cm}^{2} \mathrm{~V}^{-1} \mathrm{~s}^{-1}\right)$. On the other hand, the threshold voltage $V_{\text {th }}$ was almost the same, $-7.9 \mathrm{~V}$ for the PFBT-Au device and $-9.4 \mathrm{~V}$ for the bare-Au device.
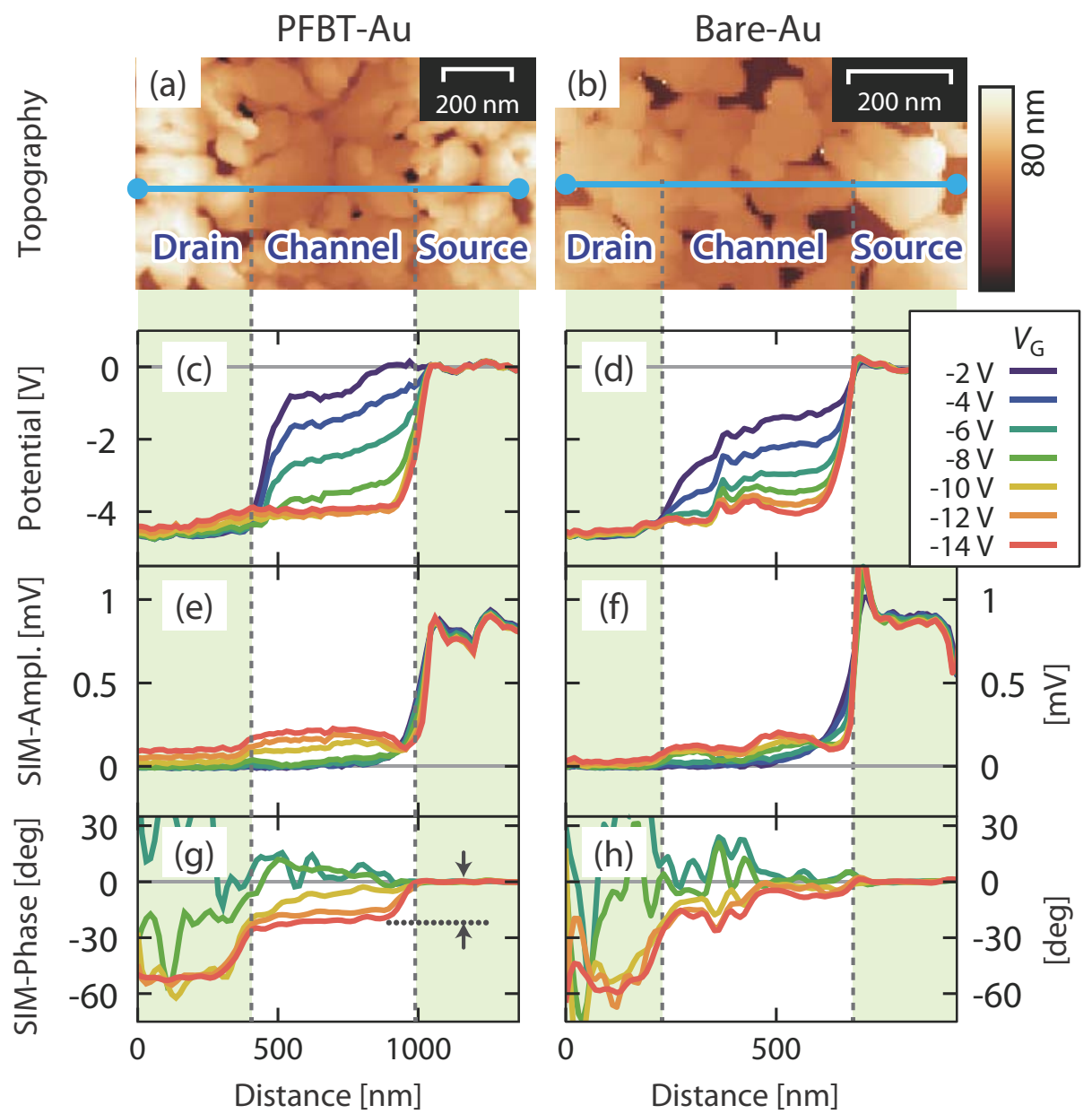

Fig. 3. FM-SIM measurements on PFBT-Au OFET (a-d) and bare-Au OFET (e-h) measured at $V_{\mathrm{D}}=$ $-5 \mathrm{~V}$ and $V_{\mathrm{G}}$ ranging from $-2 \mathrm{~V}$ to $-14 \mathrm{~V}$. (a) and (b) are topographic images of OFETs obtained before FM-SIM measurement. Profiles of (c), (d) surface potential, (e), (f) amplitude of FM-SIM signals, and (g), (h) phase of FM-SIM signals across the channel along the solid line indicated in (a) and (b) are also shown. 
The topographic images of the devices obtained by FM-AFM show that the DNTT films in both OFETs consist of grains with a feature size of 100-200 nm (see Figs. 3(a) and 3(b)). These images indicate that the SAM modification process had little influence on the surface properties of the $\mathrm{SiO}_{2}$ layer, which agrees with the fact that the thiol group of PFBT molecule links to metal surfaces. In addition, it is also consistent with the previous studies reporting the correlation between the grain size and the threshold voltage of the OFETs $[19,20]$. Therefore, the improvement in the device mobility is attributed to the change in the local electrical properties at the electrode-channel interfaces.

We recorded the FM-KFM and FM-SIM signals during scanning the tip across the channel of both OFETs while the drain bias $\left(V_{\mathrm{D}}\right)$ was set to $-5 \mathrm{~V}$ and the gate bias $\left(V_{\mathrm{G}}\right)$ was changed from $-2 \mathrm{~V}$ to $-14 \mathrm{~V}$. Figures $3(\mathrm{c})$ and $3(\mathrm{~d})$ show the surface potential profiles. We found that the surface potential profiles of both OFETs exhibited a gradual change according to $V_{\mathrm{G}}$, and eventually showed a significant potential drop at the source-channel interface in both OFETs at $V_{\mathrm{G}}<-8 \mathrm{~V}$, when the drain current began to flow as seen in Fig. 1(b). This result clearly indicated that the charge transport in both OFETs was limited at the source-channel interface. Figures 3(e) and 3(f) show the FM-SIM amplitude profiles, and Figs. 3(g) and 3(h) are the FM-SIM phase profiles. The FM-SIM amplitude profiles of both OFETs are similar; they showed a significant drop at the source-channel interface. On the other hand, we found a clear drop at the source-channel interfaces in the FM-SIM phase profiles of PFBT-Au OFET measured at $V_{\mathrm{G}}<-10 \mathrm{~V}$, while no obvious drops were observed in those of the bare-Au OFET. Considering that the variation in the FM-SIM phase signal was caused by the change in the local impedance, the result indicates that the difference in the electrical characteristics at the source-channel interface, which was not revealed from the potential profiles obtained by the KFM technique, was directly revealed by FM-SIM. We then focused on the local impedance at the sourcechannel interface. We first investigated the local impedance as a function of $V_{\mathrm{G}}$ with no applied drain bias, and then investigated the local impedance when operating the OFETs at $V_{\mathrm{D}}<0 \mathrm{~V}$. 
(a)

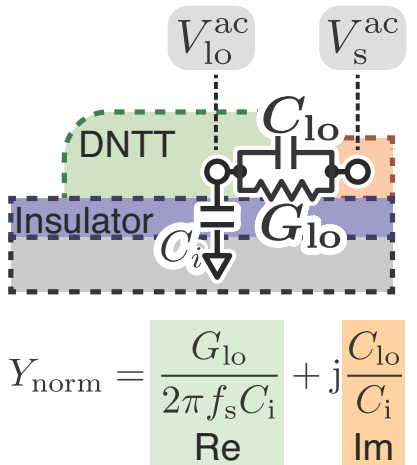

(c)

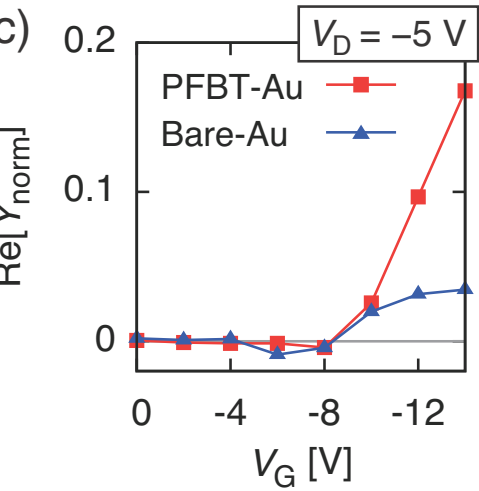

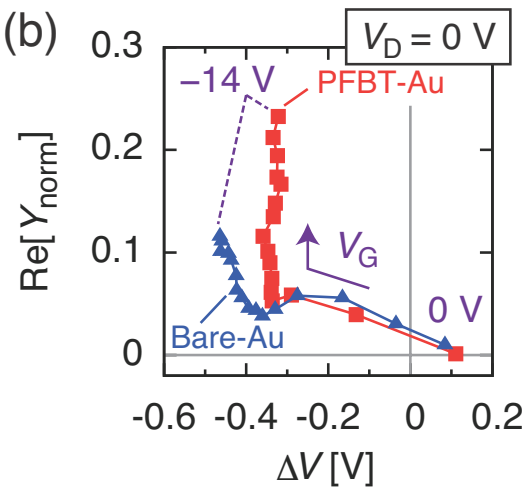

(d)

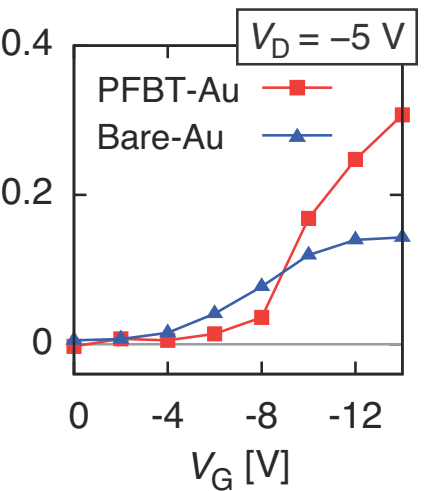

Fig. 4. (a) Schematic illustration of the sample structure and the equivalent circuit. $G_{\mathrm{lo}}$ and $C_{\mathrm{lo}}$ are the local conductance and capacitance, respectively, at the source-channel interface, and $C_{\mathrm{i}}$ is the gate capacitance. (b) Plots of the normalized conductance $\operatorname{Re}\left[Y_{\text {norm }}\right]$ as a function of the voltage drop $\Delta V$. The data were recorded while $V_{\mathrm{G}}$ was changed from $0 \mathrm{~V}$ to $-14 \mathrm{~V}$ in the direction indicated by the arrow. (c) and (d) are the plots of the normalized conductance and susceptance, respectively, as a function of $V_{\mathrm{G}}$. The square and triangle symbols represent the results of the PFBT-Au and bare-Au OFETs, respectively.

When the drain bias voltage $\left(V_{\mathrm{D}}\right)$ was not applied, the interface impedance is well modeled by the parallel circuit of the resistor and capacitor (RC-parallel) as discussed in our previous paper [14]. Therefore, we discuss in terms of the admittance (reciprocal of impedance) rather than the impedance. Using the equivalent circuit shown in Fig. 4(a), we define the normalized admittance, which is the interface admittance normalized by the effective insulator susceptance, given by

$$
Y_{\text {norm }}=\left(G_{\mathrm{lo}}+\mathrm{j} 2 \pi C_{\mathrm{lo}}\right) / 2 \pi f_{\mathrm{s}} C_{\mathrm{i}}=\mathrm{j} \gamma /(1-\gamma)
$$

where $G_{\mathrm{lo}}$ and $C_{\mathrm{lo}}$ are the interface conductance and capacitance, respectively, $C_{\mathrm{i}}$ is the insulator 
capacitance and $\gamma$ is referred to as the normalized FM-SIM signal. $\gamma$ is the complex value obtained from the FM-SIM signals as $\gamma=\alpha V_{\mathrm{lo}}^{\mathrm{ac}} \mathrm{e}^{\mathrm{j} \phi_{\mathrm{lo}}} / \alpha V_{\mathrm{s}}^{\mathrm{ac}}$. See Supplementary Information available as Appendix A for the derivation of Eq. 2 (S1) and for the justification of the parallel equivalent circuit (S2). Figure 4(b) shows the plots of the interface conductance (real part of $Y_{\text {norm }}$ ) at $V_{\mathrm{D}}=0 \mathrm{~V}$ as a function of the voltage drop $\Delta V$ at the interface, simultaneously measured by FM-KFM. As the magnitude of the negative $V_{\mathrm{G}}$ was increased in both OFETs, $\Delta V$ was shifted in the negative direction and the interface conductance was increased in a manner similar to our previous study [14]. While $\Delta V$ in PFBT-Au OFET almost saturated at $-0.35 \mathrm{~V}, \Delta V$ in the bare-Au OFET was slightly higher than that in the PFBT-Au OFET for $V_{\mathrm{G}}<-5 \mathrm{~V}$, and it even gradually shifted in the negative direction. The values of $\Delta V$ at which the interface conductance increases were about $-0.3 \mathrm{~V}$ for the PFBT-Au OFET and about $-0.4 \mathrm{~V}$ for the bare-Au OFET. As these values are correlated with the initial energy level mismatches between $E_{\mathrm{F}}$ and $E_{\mathrm{HOMO}}$, this result indicates that the initial energy level mismatch between $E_{\mathrm{F}}$ and $E_{\mathrm{HOMO}}$ was greater in the bare-Au OFET than that in the PFBT-Au OFET. Several studies showed an increase in the metal work function by the modification with the fluorinated thiol-SAM $[8,9,12]$, which is partially consistent with our result in terms of the reduction of the hole injection barrier. However, the magnitude of the reduction in the energy level mismatch in these studies varied from $0.5 \mathrm{eV}$ to $1 \mathrm{eV}$. Therefore, the reduction of the contact resistance by the SAM modification may not be fully attributed to the reduction in the energy level mismatch.

Figure 4(c) shows the interface conductance of the source-channel interfaces of the two OFETs at $V_{\mathrm{D}}=-5 \mathrm{~V}$ as a function of $V_{\mathrm{G}}$. The figure shows that the interface conductance of both OFETs started to increase at $V_{\mathrm{G}}=-8 \mathrm{~V}$. This result is in good agreement with the threshold voltage measured in Fig. 1(b), which again shows that the source-channel interface limited the device performance. This suggests that we can use the two-terminal equivalent circuit model in Fig. 4(a) despite the OFETs were in operation with a non-zero $V_{\mathrm{D}}$. We found a continuous increase in the interface conductance at $V_{\mathrm{G}}<-8 \mathrm{~V}$ in the PFBT-Au OFET, while not in the bare-Au OFET. The interface conductance by the FM-SIM measurement is not influenced by the grain boundaries around the grains of interest, but it is an intrinsic property of the metal-organic interface. This result indicates that we 
directly characterized the reduction of the contact resistance at the metal-organic interface in the OFET with the PFBT-modified electrodes. Therefore, the increase in the mobility of the OFET in Fig. 1(b) can be attributed to the reduction of the source-channel contact resistance, not to the difference in the film morphology near the electrodes.

(a) PFBT-Au

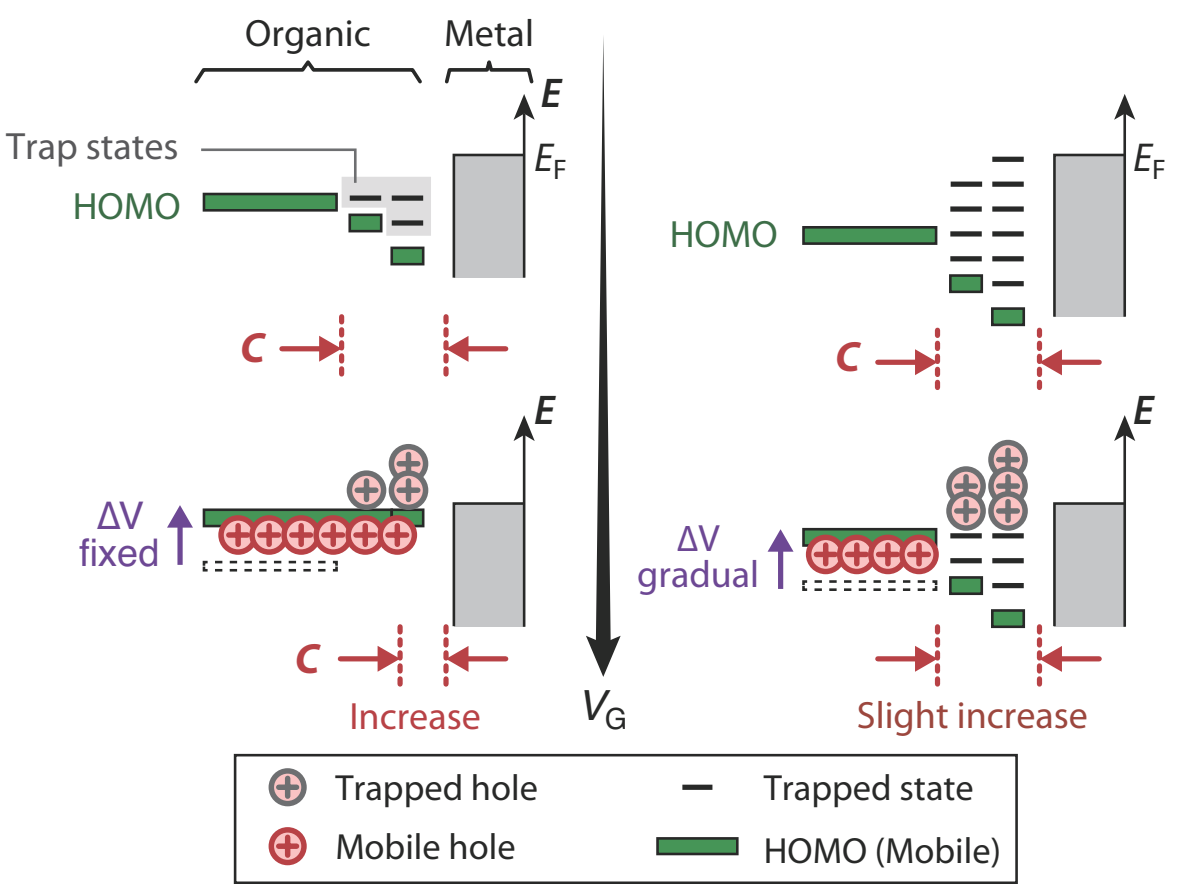

Fig. 5. Schematic energy diagram at the source-channel interfaces in PFBT-Au OFET (a) and bare-Au OFET (b), when the gate bias $\left(V_{\mathrm{G}}\right)$ was changed from $0 \mathrm{~V}$ (upper row) to a negative bias (lower row).

Figure 4(d) shows the interface susceptance which is proportional to the capacitance at the metal-organic interface at $V_{\mathrm{D}}=-5 \mathrm{~V}$ as a function of $V_{\mathrm{G}}$. It slightly increased in the bare-Au OFET as the more negative $V_{\mathrm{G}}$ was applied, while it significantly increased in the PFBT-Au OFET. As the organic film is discontinuous at the metal-organic interface, it is expected that the density of the trap sites are more in the interface than that in the channel region. The trap-rich region at the interfaces forms a depletion layer, which is often revealed by the electrical measurement as the interface capacitance [21-23]. During the carrier injection into the organic layer, the trap sites in the depletion 
layer are gradually filled and the thickness of the depletion layer is reduced. The rapid increase in the interface capacitance in the PFBT-Au OFET can be explained by this mechanism (see Fig. 5(a)). On the other hand, for the bare-Au OFET, it is expected that the amount of the trap sites at the metalorganic interface is high, and the reduction in the depletion width is limited (see Fig. 5(b)). It has been reported that the insertion of a doping layer between the electrode and the active layer in OFET reduces the thickness of the depletion layer [24]. It has also been reported that buffer layers, such as the SAM and thin oxide layer, suppress the interaction between the molecule and the metal, which reduces the trap sites at the metal-organic interface [11,25]. The reduction of the amount of the trap sites due to SAM modification is one of possible reasons for the increase in the interfacial capacitance. In order to analyze the effect of the interfacial traps on the impedance, it is desirable to measure the FM-AFM signal for more broad frequency range and analyze the result with more detailed model, for example by Nicollian and Brews [26,27], which will be subject to future studies.

\section{Conclusions}

In summary, we investigated the effect of the electrode modification by PFBT-SAM on the DNTT-OFETs using FM-SIM. The field-effect mobility of the OFET with the SAM-modified electrodes was more than twice as high as that for the OFET with bare Au electrodes. The potential profiles revealed that the electrical characteristics of the source-channel interface determine the device performances. We found a lower potential drop at the source-channel interface as well as a higher interfacial conductance in the accumulation regime for the SAM-modified OFET, which indicates the reduction in the energy level mismatch between the Fermi level of the electrode and the HOMO level of the channel layer. The reduction in the trap sites at the metal-organic interface is a possible reason for the significant increase of the interface capacitance in the SAM-modified OFET. We separately elucidated these two effects that accounted for the improvement in the device performance by the SAM-modification.

\section{Acknowledgments}

This work was supported by Grants-in-Aid for Scientific Research from the Japan Society for the Promotion of Science. 


\section{References}

[1] T. Sekitani, T. Someya, Adv. Mater. 22 (2010) 2228.

[2] R. Nakahara, M. Uno, T. Uemura, K. Takimiya, J. Takeya, Adv. Mater. 24 (2012) 5212.

[3] D.J. Gundlach, L. Zhou, J.A. Nichols, T.N. Jackson, P. V. Necliudov, M.S. Shur, J. Appl. Phys. 100 (2006) 024509.

[4] K.P. Puntambekar, P. V. Pesavento, C.D. Frisbie, Appl. Phys. Lett. 83 (2003) 5539.

[5] H. Minemawari, T. Yamada, H. Matsui, J. Tsutsumi, S. Haas, R. Chiba, R. Kumai, T. Hasegawa, Nature 475 (2011) 364.

[6] A. Valletta, A. Daami, M. Benwadih, R. Coppard, G. Fortunato, M. Rapisarda, F. Torricelli, L. Mariucci, Appl. Phys. Lett. 99 (2011) 233309.

[7] I. Kymissis, C.D. Dimitrakopoulos, S. Purushothaman, IEEE Trans. Electron. Devices 48 (2001) 1060.

[8] J.-P. Hong, A.-Y. Park, S. Lee, J. Kang, N. Shin, D.Y. Yoon, Appl. Phys. Lett. 92 (2008) 143311

[9] P. Marmont, N. Battaglini, P. Lang, G. Horowitz, J. Hwang, A. Kahn, C. Amato, P. Calas, Org. Electron. 9 (2008) 419.

[10] C.-H. Kim, H. Hlaing, J.-A. Hong, J.-H. Kim, Y. Park, M.M. Payne, J.E. Anthony, Y. Bonnassieux, G. Horowitz, I. Kymissis, Adv. Mater. Interfaces 2 (2015) 1400384.

[11] C. Bock, D. V. Pham, U. Kunze, D. Käfer, G. Witte, C. Wöll, J. Appl. Phys. 100 (2006) 114517.

[12] Z. Jia, V.W. Lee, I. Kymissis, L. Floreano, A. Verdini, A. Cossaro, A. Morgante, Phys. Rev. B $82(2010) 125457$.

[13] J. Zaumseil, K.W. Baldwin, J.A. Rogers, J. Appl. Phys. 93 (2003) 6117.

[14] T. Kimura, K. Kobayashi, H. Yamada, J. Appl. Phys. 118 (2015) 055501.

[15] D.J. Gundlach, J.E. Royer, S.K. Park, S. Subramanian, O.D. Jurchescu, B.H. Hamadani, A.J. 
Moad, R.J. Kline, L.C. Teague, O. Kirillov, C.A. Richter, J.G. Kushmerick, L.J. Richter, S.R. Parkin, T.N. Jackson, J.E. Anthony, Nat. Mater. 7 (2008) 216.

[16] S.K. Park, T.N. Jackson, J.E. Anthony, D.A. Mourey, Appl. Phys. Lett. 91 (2007) 063514.

[17] M. Nonnenmacher, M.P. O’Boyle, H.K. Wickramasinghe, Appl. Phys. Lett. 58 (1991) 2921.

[18] K. Kobayashi, H. Yamada, H. Itoh, T. Horiuchi, K. Matsushige, Rev. Sci. Instrum. 72 (2001) 4383.

[19] A. Bolognesi, M. Berliocchi, M. Manenti, A. Di Carlo, P. Lugli, K. Lmimouni, C. Dufour, IEEE Trans. Electron. Devices 51 (2004) 1997.

[20] T. Kimura, Y. Miyato, K. Kobayashi, H. Yamada, K. Matsushige, Jpn. J. Appl. Phys. 51 (2012) 08KB05.

[21] T. Miyadera, T. Minari, K. Tsukagoshi, H. Ito, Y. Aoyagi, Appl. Phys. Lett. 91 (2007) 013512.

[22] B.H. Hamadani, C. a. Richter, J.S. Suehle, D.J. Gundlach, Appl. Phys. Lett. 92 (2008) 203303

[23] P. V. Pesavento, R.J. Chesterfield, C.R. Newman, C.D. Frisbie, J. Appl. Phys. 96 (2004) 7312.

[24] T. Minari, P. Darmawan, C. Liu, Y. Li, Y. Xu, K. Tsukagoshi, Appl. Phys. Lett. 100 (2012) 093303.

[25] M. Kano, T. Minari, K. Tsukagoshi, Appl. Phys. Lett. 94 (2009) 143304.

[26] E.H. Nicollian, J.R. Brews, MOS (Metal Oxide Semiconductor) Physics and Technology, Wiley, New York, 2002.

[27] M. Petrosino and A. Rubino, Org. Electron. 12 (2011) 1159. 